\title{
Achieving Near-Exponential Diversity on Uncoded Low-Dimensional MIMO, Multi-User and Multi-Carrier Systems Without Transmitter CSI
}

\author{
Ramesh Annavajjala, Philip Orlik \\ TR2011-011 April 2011
}

\begin{abstract}
It is well-known that for single-input and single-output (SISO) narrow-band transmission on frequency-flat fading channels, uncoded communication with only receiver channel state information (Rx-CSI) leads to extremely poor reliability performance whereas transmitter CSI (TxCSI) allows us approach the reliability of an additive white gaussian noise (AWGN) channel via power control. In this paper, we propose a novel approach to achieve reliability close to the AWGN channel for uncoded transmissions on SISO frequency-flat Rayleigh fading channels without Tx-CSI. Our approach employs pseudo-random phase precoding (PRPP) of modulation symbols prior to temporal multiplexing, and joint-detection at the receiver that has polynomial complexity in the precoder size. With a precoder size of 400 binary symbols, we demonstrate that the proposed system achieves performance within $0.1 \mathrm{~dB}$ of the AWGN channel at a bit error rate of 0.00001 , and is also robust to fading correlation and channel estimation errors. Furthermore, we present extensions to multiple-user multiple-input and multiple-output (MU-MIMO) systems and wideband transmission schemes such as orthogonal frequency-division multiplexing (OFDM) and single carrier frequency-domain multiple access (SC-FDMA) systems. We show, through extensive simulations, that i) with 8- by-8 MIMO system per-stream AWGN channel reliability is achieved with 8 spatial streams and 50 channel uses, ii) for a 5 user multiple-access channel with one antenna per user and 5 antennas at the receiver, 80 channel uses eliminates fading and interference complexity while simultaneously providing a power gain of approximately $6.9 \mathrm{~dB}$, and iii) for OFDM and SC-FDMA systems with single antenna at the transmitter and two antennas at the receiver, within 0.1 and $0.3 \mathrm{~dB}$ of the matched-filter bound performance is achieved with a precoder size of 96 and 400 symbols, respectively.
\end{abstract}

IEEE Information Theory and Applications 2011

This work may not be copied or reproduced in whole or in part for any commercial purpose. Permission to copy in whole or in part without payment of fee is granted for nonprofit educational and research purposes provided that all such whole or partial copies include the following: a notice that such copying is by permission of Mitsubishi Electric Research Laboratories, Inc.; an acknowledgment of the authors and individual contributions to the work; and all applicable portions of the copyright notice. Copying, reproduction, or republishing for any other purpose shall require a license with payment of fee to Mitsubishi Electric Research Laboratories, Inc. All rights reserved. 



\title{
Achieving Near-Exponential Diversity on Uncoded Low-Dimensional MIMO, Multi-User and Multi-Carrier Systems Without Transmitter CSI
}

\author{
Ramesh Annavajjala and Philip V. Orlik \\ Digital Communications Group \\ Mitsubishi Electric Research Laboratories (MERL), Cambridge, MA, 02139, USA \\ Email: annavajjala@merl.com,porlik@merl.com
}

\begin{abstract}
It is well-known that for single-input and singleoutput (SISO) narrow-band transmission on frequency-flat fading channels, uncoded communication with only receiver channel state information (Rx-CSI) leads to extremely poor reliability performance whereas transmitter CSI (Tx-CSI) allows us approach the reliability of an additive white Gaussian noise (AWGN) channel via power control. In this paper, we propose a novel approach to achieve reliability close to the AWGN channel for uncoded transmissions on SISO frequency-flat Rayleigh fading channels without Tx-CSI. Our approach employs pseudo-random phase precoding (PRPP) of modulation symbols prior to temporal multiplexing, and joint-detection at the receiver that has polynomial complexity in the precoder size. With a precoder size of 400 binary symbols, we demonstrate that the proposed system achieves performance within $0.1 \mathrm{~dB}$ of the AWGN channel at a bit error rate of $10^{-5}$, and is also robust to fading correlation and channel estimation errors. Furthermore, we present extensions to multiple-user multiple-input and multiple-output (MU-MIMO) systems and wideband transmission schemes such as orthogonal frequency-division multiplexing (OFDM) and singlecarrier frequency-domain multiple access (SC-FDMA) systems. We show, through extensive simulations, that $i$ ) with an 8by- 8 MIMO system per-stream AWGN channel reliability is achieved with 8 spatial streams and 50 channel uses, $i i$ ) for a 5 user multiple-access channel with one antenna per user and 5 antennas at the receiver, 80 channel uses eliminates fading and interference completely while simultaneously providing a power gain of approximately $6.9 \mathrm{~dB}$, and $i i i$ ) for OFDM and SC-FDMA systems with single antenna at the transmitter and two antennas at the receiver, within 0.1 and $0.3 \mathrm{~dB}$ of the matched-filter bound performance is achieved with a precoder size of 96 and 400 symbols, respectively.
\end{abstract}

Index Terms-Single-antenna transmission, pseudo-random phase precoding, multi-user MIMO, matched-filter bound, uncoded multi-carrier systems, large-dimensional detection.

\section{INTRODUCTION}

Reliable communication over fading and dispersive wireless channels is an important area of research [1]. For a single-input and single-output (SISO) point-to-point link on frequency-flat fading channels, it is well-known that uncoded transmission without transmitter channel state information (Tx-CSI) has an extremely poor reliability performance [2]. With receiver CSI (Rx-CSI) only, the average uncoded probability of error on SISO fading links decays inversely with the average received signal-to-noise ratio (SNR). This is in contrast with exponential decay on additive white Gaussian noise (AWGN) channel [3]. With Tx-CSI, on the other hand, it is possible to approach the AWGN performance via temporal power control [4], [5].
Spatial diversity, via multiple antennas at the receiver, is an attractive option to improve the reliability of single antenna transmissions [6]. However, due to size and cost constraints, it may not be possible to increase the number of antennas beyond a certain limit. Channel coding, using resources in time and/or frequency, is also a well-known technique to mitigate the effects of fading [7], [8]. The coding gain and the diversity achieved against fading strongly depend on the nature of the channel and the properties of the channel code employed such as code rate, codeword length, constraint length (i.e., free distance), and decoder complexity [7].

In the context of detection of multiple data streams over multiple-input and multiple-output (MIMO) wireless channels with a large number of antennas at the transmitter and the receiver, the authors in [9] proposed a sub-optimum receiver and showed that it achieves near exponential diversity per stream as the number of streams, and the operating SNR, go to infinity [10]. This algorithm, termed likelihood ascent search (LAS), has its roots in image processing [11], [12], [13] and multiuser detection in code-division multiple access systems [14], [15], and offers excellent performance, close to maximum likelihood detection (MLD), but with per-bit complexity that grows polynomially with the number of transmitter antennas [9]. Unfortunately, the LAS algorithms in [9] and [15] that are applied to traditional multi-stream MIMO systems with a large number transmit and receive antennas are not directly applicable to a SISO system.

In this paper, we propose a novel approach to achieve reliability close to the AWGN channel for uncoded transmissions on frequency-flat SISO Rayleigh fading channels without TxCSI. The proposed approach employs pseudo-random phase precoding (PRPP) of modulation symbols prior to temporal multiplexing, thereby converting a SISO channel into a virtual MIMO channel. With the PRPP operating on 400 binary symbols over temporarily uncorrelated channels, we demonstrate that the proposed system with a sequential LAS receiver [9] achieves performance within $0.1 \mathrm{~dB}$ of the AWGN channel at a bit error rate of $10^{-5}$. We also show that the proposed system is robust to fading correlation as well as channel estimation errors at the receiver, and extends naturally to wideband transmission formats such as orthogonal frequency-division multiplexing (OFDM) [16], single-carrier frequency-domain multiple access (SC-FDMA) [16], and multi-user MIMO systems [17].

In [15], is was shown that for a large number of transmitter 
and receiver antennas, the per-stream reliability performance with the LAS algorithm approaches the AWGN channel performance. Using extensive simulation results, we show that our proposed PRPP approach when applied to uncoded singleuser MIMO system with spatial multiplexing and multi-user MIMO system with non-cooperating users also yields nearexponential diversity. Additionally, when the PRPP is applied to uncoded single-user OFDM and SC-FDMA systems with two receiver antennas yields performance within 0.1 and 0.3 $\mathrm{dB}$, respectively, of the matched-filter bound (MFB) [18], [19], [20].

We note that, similar to our PRPP approach, the diversity transform (DRT) in [21] also tries to improve the reliability of uncoded transmission over frequency-flat channels via linear transformations. Unlike the pseudo-random phasors as the precoder matrix entries in our paper, [21] restricts DRT to orthonormal matrices. More importantly, while restricted to single-user SISO systems, optimal DRT matrices in [21] are channel specific. Using signal-space diversity and multidimensional lattice constellations, the authors in [22] provide near exponential diversity at a higher demodulator complexity on SISO fading channels. On the other, the proposed PRPP approach yields AWGN-like performance even with binary signaling. In [23], the authors propose a random phase precoder for diversity improvement with multiple transmitter antennas over quasi-static fading channels. With two transmitter antennas, [23] shows an improvement over Alamouti scheme [16] only when the receiver CSI is imperfect. Moreover, with a large number of antennas the approach in [23] converts quasi-static fading into independent fading without actually eliminating the impact of fading.

The rest of this paper is organized as follows. In Section II, we present the system model. Our proposed temporal multiplexing with PRPP and the corresponding detection techniques are detailed in Section III. Simulation results on the performance of the PRPP-based SISO system are presented in Section IV. Extensions to block fading channels, single-user MIMO, multi-user MIMO, and OFDM and SC-FDMA systems are detailed in Sections V, VI, VII, and VIII, respectively. We conclude this work in Section IX.

\section{Single User SISO System Model}

We consider a point-to-point communication system with a single antenna at the transmitter and a single antenna at the receiver. The channel between the transmitter and the receiver at the $n$th channel use is modeled as a frequency-flat and slowly varying random variable (r.v) $h_{n}$. The transmitted signal at time $n$ is $x_{n}$ with mean $E\left[x_{n}\right]=0$ and variance $E\left[\left|x_{n}\right|^{2}\right]=\sigma_{x}^{2}$, and the corresponding received signal $y_{n}$ is given by

$$
y_{n}=h_{n} x_{n}+w_{n}
$$

where $w_{n}$ is a zero-mean complex-Gaussian r.v with variance $E\left[\left|w_{n}\right|^{2}\right]=\sigma_{w}^{2}$. With $N$ uses of the channel, (1) in matrixvector notation is expressed as

$$
\mathbf{y}=\mathbf{H x}+\mathbf{w}
$$

where $\mathbf{y}=\left[y_{1}, y_{2}, \ldots, y_{N}\right]^{T}, \mathbf{x}=\left[x_{1}, x_{2}, \ldots, x_{N}\right]^{T}, \mathbf{H}=$ $\operatorname{Diag}\left(h_{1}, \ldots, h_{N}\right), \mathbf{w}=\left[w_{1}, w_{2}, \ldots, w_{N}\right]^{T}$, and $T$ denotes the transpose operation.
With each $x_{n}$ drawn independently from a constellation $\mathcal{S}$ with $M$ points, the maximum likelihood detector (MLD) of $\mathbf{x}$ has the following well-known form

$$
\begin{aligned}
\widehat{\mathbf{x}}_{M L} & =\arg \min _{\mathbf{x} \in \mathcal{S}^{N}}\|\mathbf{y}-\mathbf{H} \mathbf{x}\|^{2} \\
& =\arg \min _{\mathbf{x} \in \mathcal{S}^{N}} \sum_{n=1}^{N}\left|y_{n}-h_{n} x_{n}\right|^{2} \\
& =\left[\widehat{x}_{1, M L}, \cdots, \widehat{x}_{N, M L}\right]^{T}
\end{aligned}
$$

where $\widehat{x}_{n, M L}=\arg \min _{x_{n} \in \mathcal{S}}\left|y_{n}-h_{n} x_{n}\right|^{2}$. That is, joint ML detection of $N$ symbols is equivalent to symbol-by-symbol ML detection. However, the main limitation of the above approach is that the reliability per channel use, as measured by the average bit error rate (BER), is extremely poor. As an example, with $M=2$ and $\mathcal{S}=\left\{+\sigma_{x},-\sigma_{x}\right\}$ with equal probability, the average BER on uncorrelated Rayleigh fading channels (i.e., when $h_{n}$ is a zero-mean complex-Gaussian r.v with variance $E\left[\left|h_{n}\right|^{2}\right]=\sigma_{h}^{2}$ ) is [3]

$$
\begin{aligned}
\bar{P}_{b} & =\frac{1}{N} \sum_{n=1}^{N} \operatorname{Prob}\left(\widehat{x}_{n} \neq x_{n}\right)=\frac{1}{2}\left(1-\sqrt{\frac{\sigma_{x}^{2} \sigma_{h}^{2}}{\sigma_{w}^{2}+\sigma_{x}^{2} \sigma_{h}^{2}}}\right) \\
& \approx \frac{1}{4 \mathrm{SNR}} \text { for high SNR, }
\end{aligned}
$$

where SNR $=\sigma_{x}^{2} \sigma_{h}^{2} / \sigma_{w}^{2}$ is the average received SNR per symbol. (4) must be contrasted against the performance on an AWGN channel [2] (i.e., when $h_{n}=1$ )

$$
\bar{P}_{b}^{A W G N}=\frac{1}{N} \sum_{n=1}^{N} \operatorname{Prob}\left(\widehat{x}_{n} \neq x_{n}\right)=Q(\sqrt{2 \mathrm{SNR}})
$$

where $Q(x)=(1 / \sqrt{2 \pi}) \int_{x}^{\infty} \exp \left(-t^{2} / 2\right) d t$ is the complimentary cumulative distribution function of the standard Gaussian r.v. Note that (4) has inverse linear relationship with the average received SNR, whereas (5) decays exponentially with SNR. In the following sections, we describe novel approaches to uncoded information transmission over SISO, MIMO, multiuser and multi-carrier fading channels that achieve the perstream reliability performance very close to (5) without rate loss and transmitter CSI.

\section{TEMPORAL MUltipleXING With PRPP}

The proposed PRPP transmitter and receiver with temporal multiplexing are illustrated in Figs. 1a and 1b, respectively. The complex-valued information symbol sequence is denoted by $\mathcal{U}=\left\{u_{1}, u_{2}, \ldots\right\}$, where $u_{i}$ is drawn independently from a constellation $\mathcal{S}$ containing $M$ points with $E\left[u_{i}\right]=0$ and $E\left[\left|u_{i}\right|^{2}\right]=\sigma_{u}^{2}$. For a given precoder size $N$, the information symbol vector at the input of the PRPP is denoted by $\mathbf{u}=$ $\left[u_{1}, u_{2}, \ldots, u_{N}\right]^{T}$. The output of the precoder is denoted by $\mathbf{x}$ and is simply $\mathbf{x}=\mathbb{P} \mathbf{u}$. The $(m, n)$ th element of $\mathbb{P}=\left[p_{m, n}\right]$ is $p_{m, n}=e^{j \theta_{m, n}} / \sqrt{N}, m=1, \ldots, N, n=1, \ldots, N$, where $\theta_{m, n}$ is pseudo-randomly generated by a seed shared between the transmitter and the receiver. The $m$ th element $x_{m}$ of $\mathbf{x}$ is

$$
x_{m}=\frac{1}{\sqrt{N}} \sum_{n=1}^{N} e^{j \theta_{m, n}} u_{n}
$$




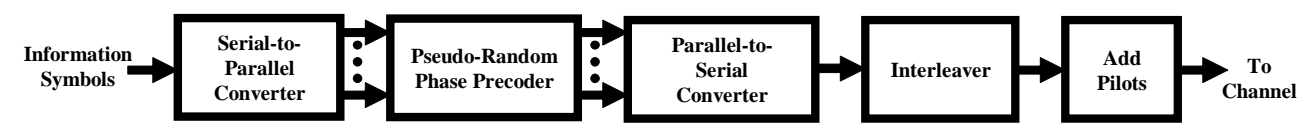

(a)

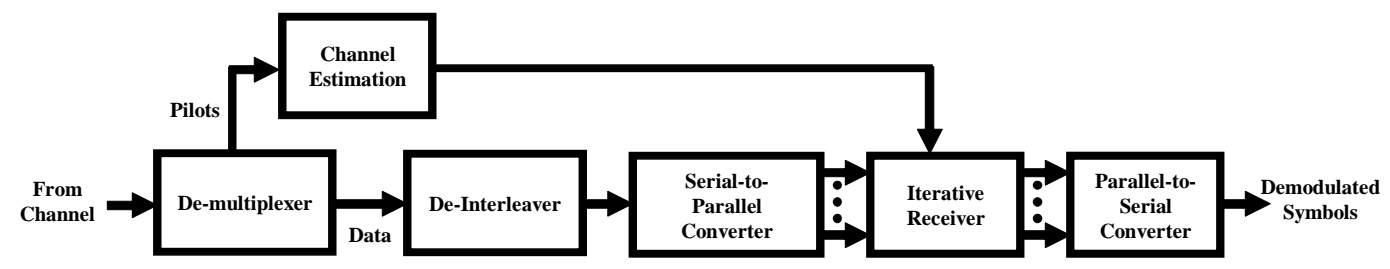

(b)

Fig. 1: Proposed pseudo-random phase precoder (PRPP) transmitter, Fig. 1a, and receiver, Fig. 1 b.

With uniformly distributed $\theta_{m, n}$ in $[-\pi,+\pi)$, a reasonable assumption for a long pseudo-noise sequence, and due to the independence of $\theta_{m, n}$ 's and $u_{n}$ 's, we have

$$
\begin{aligned}
E\left[x_{m}\right] & =\frac{1}{\sqrt{N}} \sum_{n=1}^{N} E\left[e^{j \theta_{m, n}}\right] E\left[u_{n}\right]=0 \\
\text { and } E\left[x_{m} x_{l}^{*}\right] & =\frac{1}{N} \sum_{n=1}^{N} \sum_{q=1}^{N} E\left[e^{j \theta_{m, n}} e^{-j \theta_{l, q}}\right] E\left[u_{n} u_{q}^{*}\right] \\
& =\frac{\sigma_{u}^{2}}{N} \sum_{n=1}^{N} \sum_{q=1}^{N} E\left[e^{j \theta_{m, n}} e^{-j \theta_{l, q}}\right] \delta_{n-q} \\
& =\frac{\sigma_{u}^{2}}{N} \sum_{n=1}^{N} E\left[e^{j \theta_{m, n}} e^{-j \theta_{l, n}}\right] \\
& =\sigma_{u}^{2} \delta_{m-l}
\end{aligned}
$$

where $\delta_{k}$ is the Kronecker delta function that evaluates to one when $k=0$ and zero otherwise. That is, with uncorrelated input sequence, the PRPP produces uncorrelated output sequence with the same average power as that of the input sequence that is applied to it.

Instead of transmitting the original sequence $\mathcal{U}$, we transmit the sequence $\mathcal{X}=\left\{x_{1}, x_{2}, \ldots\right\}$ produced by the PRPP. Since each $x_{n}$ goes through the flat-fading channel, as per the inputoutput relationship in (1), upon collecting complex-valued received symbols over $N$ channel uses, from (2), we have

$$
\begin{aligned}
\mathbf{y} & =\mathbf{H} \mathbf{x}+\mathbf{w}=\mathbf{H P} \mathbf{u}+\mathbf{w} \\
& =\mathbf{G u}+\mathbf{w},
\end{aligned}
$$

where $\mathbf{G}=\left[g_{m, n}\right]=\operatorname{Diag}\left(h_{1}, \ldots, h_{N}\right) \mathbb{P}$ is an $N-$ by $-N$ matrix whose $(m, n)$ th element, $g_{m, n}$, is given by

$$
g_{m, n}=h_{m} e^{j \theta_{m, n}} / \sqrt{N}
$$

Clearly, due to the independence of $h_{m}$ 's and $\theta_{m, n}$ 's, we have

$$
\begin{aligned}
E\left[g_{m, n}\right] & =E\left[h_{m}\right] E\left[e^{j \theta_{m, n}}\right] / \sqrt{N}=0 \\
\text { and } E\left[g_{m, n} g_{p, q}^{*}\right] & =E\left[h_{m} h_{p}^{*}\right] E\left[e^{j \theta_{m, n}} e^{-j \theta_{p, q}}\right] / N \\
& =\left(\sigma_{h}^{2} / N\right) \delta_{m-p} \delta_{n-q} .
\end{aligned}
$$

That is, from (9)-(12), we conclude that the PRPP applied to $N$ symbols with single-antenna transmission produces an $N-$ by $-N$ virtual MIMO system with $N$ virtual spatial streams and uncorrelated channels between each transmitreceive antenna pair. It is worth mentioning that the virtual MIMO system in (9) is different from the conventional MIMO system in the following respects:

- The effective rate of (9) is one symbol per channel use.

- Although all the elements of $\mathbf{G}$ are uncorrelated, the amplitudes of elements in a given row of $\mathbf{G}$ are identical. That is, $\left|g_{m, n}\right|=\left|h_{m}\right|$ for $n=1, \ldots, N$ showing the lack of rich scattering in $\mathbf{G}$.

- Any fixed unitary precoder $\mathbf{U}$ would satisfy the power constraint in (8). That is, with $\mathbf{x}=\mathbf{U u}$, we have $E\left[\mathbf{x}^{\dagger} \mathbf{x}\right]=E\left[\mathbf{u}^{\dagger} \mathbf{U}^{\dagger} \mathbf{U} \mathbf{u}\right]=E\left[\mathbf{u}^{\dagger} \mathbf{u}\right]$. However, such $\mathbf{U}$, in general, does not satisfy (11) and (12).

\section{A. Detection of PRPP Temporal-Multiplexing Systems}

For a PRPP of size $N$, exact MLD of $\mathbf{u}$ in (9) has complexity proportional to $M^{N}$, which is prohibitively expensive even for binary modulations $(M=2)$ and a precoder operating on $N=10$ symbols. Near-ML sphere-decoding algorithms can be applied to (9) to detect $\mathbf{u}$. However, they too have complexity exponential in $N$, and the exact complexity is a function of the operating SNR as well [24]. Sub-optimal MIMO detection algorithms such as linear zero-forcing ( $\mathrm{ZF}$ ) and minimum mean-square error (MMSE) receivers have complexity of the order of $N^{3}$ (since they require a matrix inversion) but provide a per-symbol diversity of only one [17]. The near-ML LAS algorithm in [9] essentially performs an $N$-dimensional search to arrive at a fixed point $\mathbf{u}$ that no longer improves the likelihood (conditioned on the channel and the received vector), and has a complexity proportional to $N^{3}$ (i.e., comparable to a linear MMSE receiver). Thus, we propose to use a sequential version of LAS (SLAS) [9] which is described below for binary modulations.

1) We denote by $\widehat{\mathbf{G}}=\operatorname{Diag}\left(\widehat{h}_{1}, \ldots, \widehat{h}_{N}\right) \times \mathbb{P}$ an estimate of $\mathbf{G}$ at the receiver, where $\widehat{h}_{n}$ is the estimate of $h_{n}$. Assuming binary modulation, the log-likelihood function of $\mathbf{u}$, after ignoring constants that are not a function of $\mathbf{u}$, can be written as

$$
\begin{aligned}
\Lambda(\mathbf{u}) & =-\|\mathbf{y}-\widehat{\mathbf{G}} \mathbf{u}\|^{2} \\
& =2 \operatorname{Real}\left\{\mathbf{u}^{T} \widehat{\mathbf{G}}^{\dagger} \mathbf{y}\right\}-\mathbf{y}^{\dagger} \mathbf{y}-\mathbf{u}^{T} \widehat{\mathbf{G}}^{\dagger} \widehat{\mathbf{G}} \mathbf{u} \\
& =\mathbf{u}^{T} \mathbf{y}_{\text {eff }}-\mathbf{u}^{T} \mathbf{G}_{\text {eff }} \mathbf{u},
\end{aligned}
$$


where we have ignored the $\mathbf{y}^{\dagger} \mathbf{y}$ term as it is not a function of $\mathbf{u}$ and defined $\mathbf{y}_{\text {eff }}=2$ Real $\left\{\widehat{\mathbf{G}}^{\dagger} \mathbf{y}\right\}$ and $\mathbf{G}_{\text {eff }}=\widehat{\mathbf{G}}^{\dagger} \widehat{\mathbf{G}}$.

2) The number of iterations is set to $N_{\text {lter }}$. We denote by $\mathbf{u}(k)=\left[u_{1}(k), \ldots, u_{N}(k)\right]$ an estimate of $\mathbf{u}$ at the end of iteration $k$. Thus, the initial vector at the start of SLAS iteration is denoted by $\mathbf{u}(0)$, and is set to

$$
\begin{aligned}
\mathbf{u}(0)= & \operatorname{sign}\left(\operatorname{Real}\left\{\left[\sigma_{w}^{2} \mathbf{I}_{N}+\widehat{\mathbf{G}}^{\dagger} \widehat{\mathbf{G}}\right]^{-1} \widehat{\mathbf{G}}^{\dagger} \mathbf{y}\right\}\right) \\
= & \operatorname{sign}\left(\operatorname { R e a l } \left\{\left[\sigma_{w}^{2} \mathbf{I}_{N}+\sum_{n=1}^{N}\left|\widehat{h}_{n}\right|^{2} \mathbf{p}_{n}^{\dagger} \mathbf{p}_{n}\right]^{-1}\right.\right. \\
& \left.\left.\times \widehat{\mathbf{G}}^{\dagger} \mathbf{y}\right\}\right)
\end{aligned}
$$

which is a linear MMSE estimate of $\mathbf{u}$. In (14), $\mathbf{p}_{j}$ is the $j$ th row of $\mathbb{P}$ and $\mathbf{I}_{N}$ is an $N$-by- $N$ identity matrix.

Since the transmitter and the receiver share the precoder $\mathbb{P}$, the receiver pre-computes the $N$ outer products $\mathbf{p}_{j}^{\dagger} \mathbf{p}_{j}, j=$ $1, \ldots, N$. Also, upon using matrix inversion lemma [25], the inverse in (14) can be computed efficiently. If $\sigma_{w}^{2}$ is not known to the receiver accurately, appropriate diagonal loading can be employed [26].

3) Define

$$
\begin{aligned}
\mathbf{G}_{\text {Real }} & =2 \operatorname{Real}\left\{\mathbf{G}_{\text {eff }}\right\} \\
\text { and } \mathbf{g}(k) & =\mathbf{y}_{\text {eff }}-2 \operatorname{Real}\left\{\mathbf{G}_{\text {eff }}\right\} \mathbf{u}(k) \\
& =\left[g_{1}(k), \ldots, g_{N}(k)\right]^{T} .
\end{aligned}
$$

The $j$ th symbol in the $(k+1)$ th iteration is updated as

$$
u_{j}(k+1)=\left\{\begin{array}{cc}
+1 & \text { if } u_{j}(k)=-1 \text { and } \\
& g_{j}(k)>\left|\mathbf{G}_{\text {Real }}(k, k)\right| \\
-1 & \text { if } u_{j}(k)=+1 \text { and } \\
& g_{j}(k)<-\left|\mathbf{G}_{\text {Real }}(k, k)\right| \\
u_{j}(k) & \text { otherwise }
\end{array}\right.
$$

In (17), $\mathbf{G}_{\text {Real }}(l, m)$ is the $(l, m)$ th entry of $\mathbf{G}_{\text {Real }}$ in (15).

4) At the end of $N_{\text {lter }}$ iterations, $\left[u_{1}\left(N_{\text {lter }}\right), \ldots, u_{N}\left(N_{\text {lter }}\right)\right]^{T}$ is our estimate of $\mathbf{u}$.

The PRPP transmitter coupled with the above described SLAS receiver algorithm and with the linear MMSE solution (14) as the initial vector is referred to as PRPP-MMSE-SLAS. At the end of $N_{\text {lter }}$, the average BER of PRPP-MMSE-SLAS system is computed as

$$
\bar{P}_{b}^{P R P P}\left(N, N_{\text {lter }}\right)=\frac{1}{N} \sum_{n=1}^{N} \operatorname{Prob}\left(u_{n}\left(N_{\text {lter }}\right) \neq u_{n}\right) .
$$

\section{Simulation Results: SiSO System With CONTINUOUS FADING}

In this section, we present some simulation results on the performance of the proposed PRPP transmission with MMSESLAS receiver. In all the simulations, we focus on binary modulation and the number of iterations in the MMSE-SLAS receiver is set to $N_{\text {lter }}=5$. With small precoder sizes, Fig. 2a compares the average BER performance of a system without PRPP and with per-symbol MLD (denoted as NoPRPP-MLD) against the PRPP transmission with both MMSE and MMSE-SLAS receivers. In Fig. 2a we set the precoder size $N \in\{10,20,30,40,50\}$ modulation symbols. The channel is assumed to undergo temporally uncorrelated Rayleigh fading. At lower average received SNR, the PRPP transmission with MMSE receiver is found to be inferior to the No-PRPP-MLD system whereas the MMSE receiver has an advantage of around $5 \mathrm{~dB}$ over the No-PRPP-MLD system in the high SNR regime. However, the SLAS receiver that uses the MMSE estimate as the initial vector provides an excellent performance over both the No-PRPP-MLD and PRPP-MMSE systems. At a BER of $10^{-4}$, and as the precoder size increases from 10 to 50 symbols, the MMSE-SLAS receiver exploits additional temporal diversity and provides more than $15 \mathrm{~dB}$ gain over both the No-PRPP-MLD and PRPP-MMSE systems.

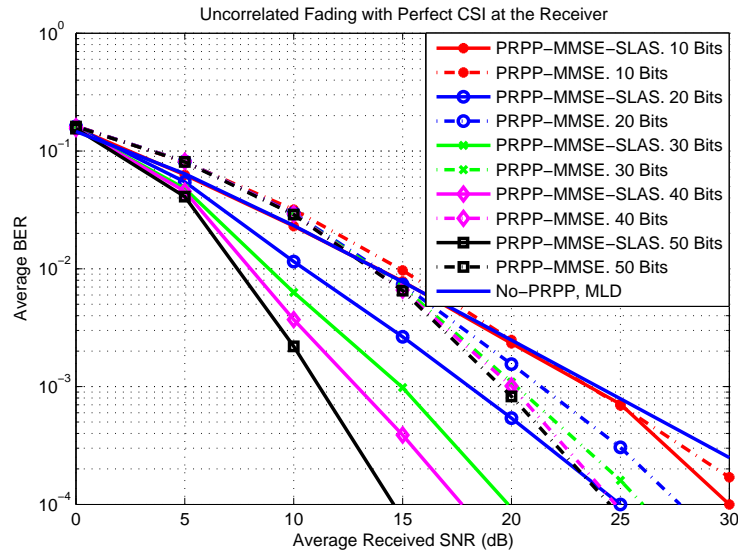

(a)

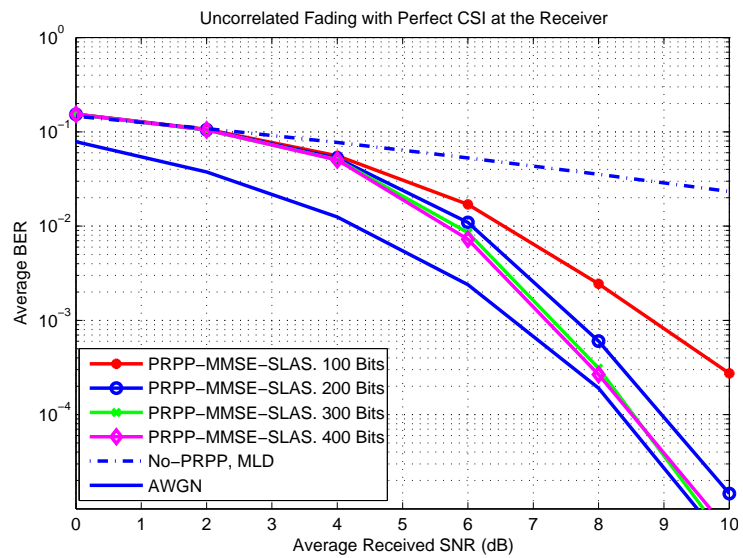

(b)

Fig. 2: Performance of PRPP on SISO fading channels. Fig. 2a shows performance comparison for smaller precoder sizes. Fig. $2 \mathrm{~b}$ shows performance comparison for moderate precoder sizes.

In Fig. $2 b$ the performance of PRPP-MMSE-SLAS system is compared against the No-PRPP-MLD receiver for precoder sizes $N \in\{100,200,300,400\}$ symbols. Also shown in Fig. $2 \mathrm{~b}$ is the performance of a SISO AWGN link whose average BER is $Q(\sqrt{2 \mathrm{SNR}})$. Similar to Fig. 2a, we have assumed a temporarily uncorrelated Rayleigh fading channel with perfect CSI at the receiver. It is interesting to note that the performance of MMSE-SLAS receiver approaches that of the 
AWGN system, even for moderate precoder sizes of 300 to 400 symbols. At an error rate of $10^{-5}$, the AWGN system requires an SNR of $9.6 \mathrm{~dB}$ whereas the proposed system with MMSESLAS receiver requires an SNR of $9.7 \mathrm{~dB}$ with 400 symbols. That is, the proposed system has near-exponential diversity and is within $0.1 \mathrm{~dB}$ of the AWGN system performance.

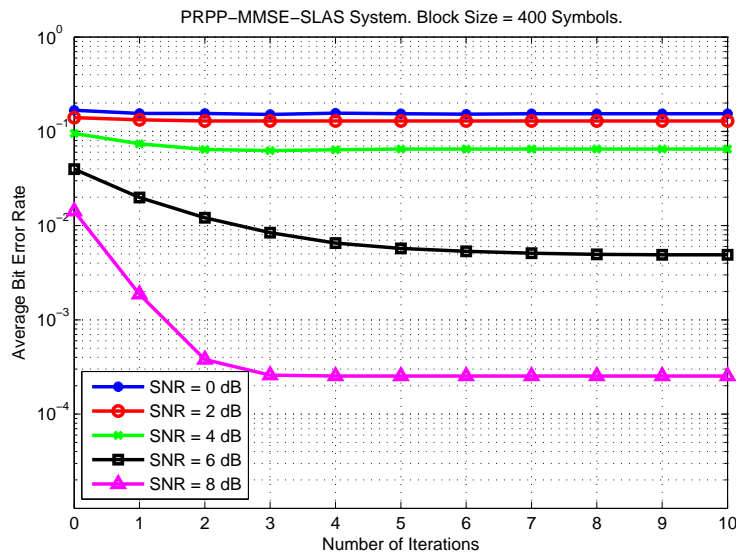

(a)

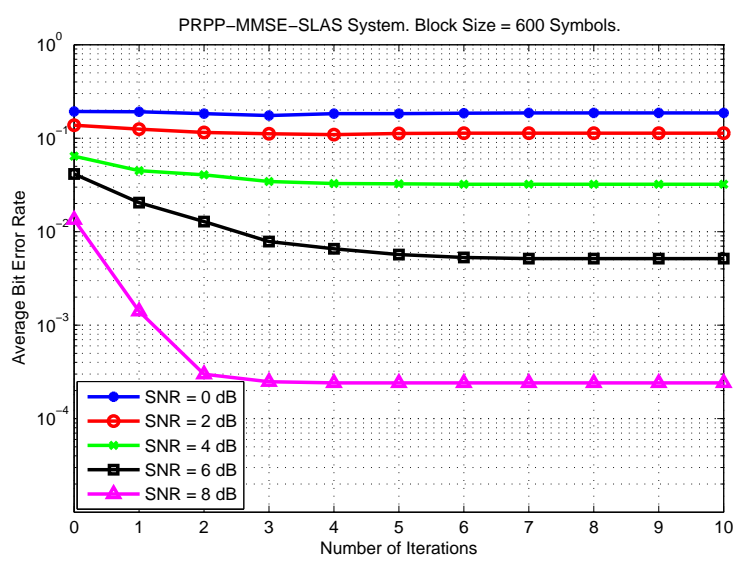

(b)

Fig. 3: BER performance as a function of the number of iterations on uncorrelated Rayleigh fading channels. The block size is 400 symbols in Fig. 3a whereas it is 600 symbols in Fig. 3b. The number of iterations $=0$ corresponds to the performance of linear MMSE receiver.

The impact of the number of iterations in the MMSE-SLAS receiver on the BER performance is studied in Figs. 3a and $3 \mathrm{~b}$ with precoder sizes of 400 and 600 symbols, respectively. From Fig. 3, we conclude that 5 iterations are sufficient over the SNRs of practical interest.

The impact of channel correlation on the performance of the proposed PRPP-MMSE-SLAS system is investigated in Fig. 4. Here, we set the precoder size to 400 modulation symbols and consider a time-correlated Rayleigh fading channel. For simplicity, in Fig. 4a, the channel correlation between two adjacent symbols in time is parameterized by $\rho, 0<\rho<1$, and the correlation between complex-valued channels at two time instances $n$ and $k$ is set to $\rho^{|n-k|}$. The average BER is plotted for various values of $\rho \in\{0.1,0.3,0.6,0.9\}$. Note that the No-PRPP-MLD performance is not affected by the channel correlation as it performs symbol-by-symbol detection. From

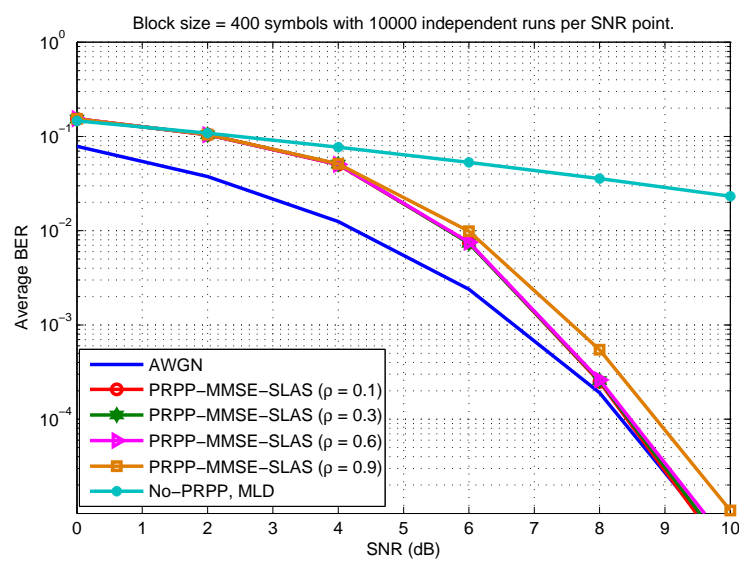

(a)

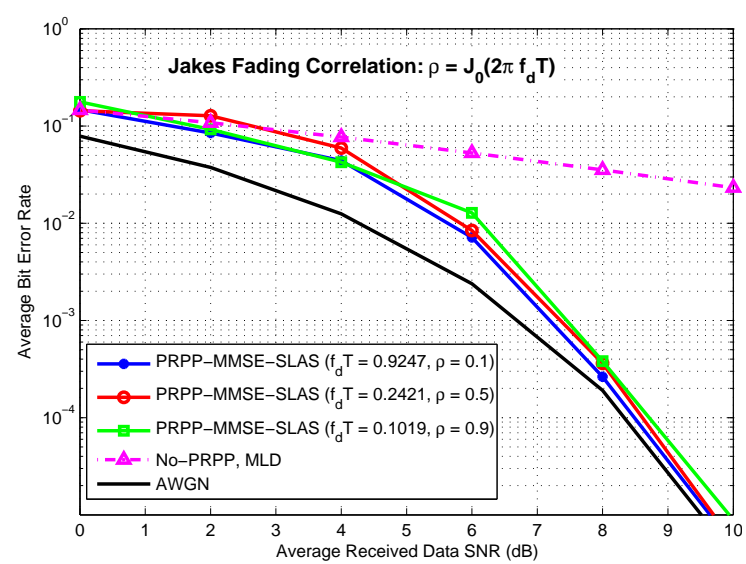

(b)

Fig. 4: Impact of channel correlation on the performance of a PRPP system with a precoder size of 400 symbols. Fig. 4a assumes an exponential time-correlation model whereas Fig. 4b considers Jakes fading model.

Fig. 4a we conclude that system performance is insensitive to channel correlations as high as $\rho=0.6$. In fact, even for $\rho$ as high as 0.9 the performance gap between the PRPP-MMSESLAS receiver and the AWGN system is as small as $0.4 \mathrm{~dB}$.

\section{PRPP FOR BLOCK FADING CHANNELS}

We now describe the application of PRPP on block fading channels. In our block fading channel model, the channel remains constant over $K$ channel uses and varies independently from block to block. The appropriate choice of $K$ depends on the channel coherence time for time-varying channels and the channel coherence bandwidth for frequency-selective channels.

As illustrated in Fig. 5a, the proposed PRPP idea with a precoder size of $N$ symbols can be applied over a block fading channel with $K$ symbols per fading block by interleaving each of the $N$ symbols within a PRPP block across $N$ fading blocks. The resulting delay with this approach, due to interleaving and de-interleaving, is $2 N K$. Each fading block of $K$ symbols is composed of $N_{D}$ data symbols and $N_{P}=K-N_{D}$ pilot symbols for channel estimation. The received signal on the $k$ th fading block during the $N_{P}$ channel uses is given by

$$
y_{k}[p]=\beta h_{k}+w_{k}[p], \quad p=1, \ldots, N_{P},
$$




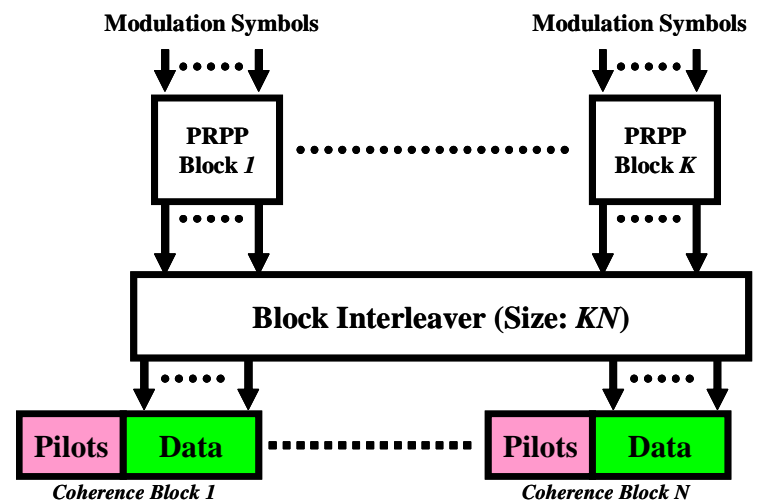

(a)

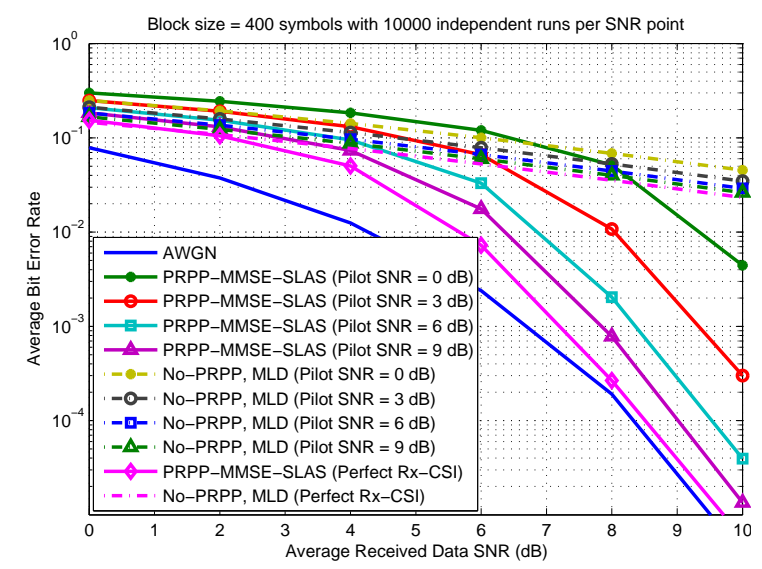

(b)

Fig. 5: PRPP on block fading channels. Fig. 5a describes the system model, whereas Fig. 5b shows the reliability performance as a function of channel estimation accuracy.

where $\beta^{2} / \sigma_{w}^{2}$ denote the average pilot SNR relative to the data SNR. The channel estimate for the $k$ th fading block is then given by

$$
\widehat{h}_{k}=\frac{1}{\beta N_{P}} \sum_{p=1}^{N_{P}} y_{k}[p]=h_{k}+\frac{1}{\beta N_{P}} \sum_{p=1}^{N_{P}} w_{k}[p] .
$$

It is easy to see that the mean-square error (MSE) between the true channel $h_{k}$ and the estimate $\widehat{h}_{k}$ is

$$
E\left[\left|h_{k}-\widehat{h}_{k}\right|^{2}\right]=\left(\frac{N_{P} \beta^{2}}{\sigma_{w}^{2}}\right)^{-1} .
$$

The impact of channel estimation errors on the performance of the PRPP-MMSE-SLAS system over block fading channels is plotted in Fig. 5b. For simplicity, the "Pilot SNR" in Fig. 5b is defined as the ratio between the average received pilot SNR and the average per-symbol data SNR, and is equal to $N_{P} \beta^{2} / \sigma_{x}^{2}$. The precoder size is set to 400 binary symbols, and the average BER performance of PRPP-MMSE-SLAS is evaluated for Pilot SNR in $\{0,3,6,9\} \mathrm{dB}$. At an average BER of $10^{-4}$, the degradation in average received SNR, compared with the performance on an AWGN channel, is approximately $1.2 \mathrm{~dB}$ when the Pilot SNR is $6 \mathrm{~dB}$. When the Pilot SNR is increased by $3 \mathrm{~dB}$ (either by doubling the pilot power $\beta^{2}$ or the number of pilots $N_{P}$ ), to $9 \mathrm{~dB}$, the penalty due to imperfect channel estimation is within $0.4 \mathrm{~dB}$ for the average BER ranging from $10^{-4}$ to $10^{-5}$.

\section{PRPP FOR SINGLE-USER MIMO SySTEMS}

Fig. 6a describes a MIMO system equipped with $N_{S}$ streams, $N_{T}$ transmit antennas and $N_{R}$ receive antennas. The transmitter precoder for channel use $n$ is denoted by the $N_{T^{-}}$ by- $N_{S}$ matrix $\mathbf{Q}_{n}$. Note that $\mathbf{Q}_{n}$ need not be a function of Tx-CSI. As an example, Fig. 6a could describe a spatialmultiplexing system with $N_{S}=N_{T}$ and $\mathbf{Q}_{n}=\mathbf{I}_{N_{T}}$.

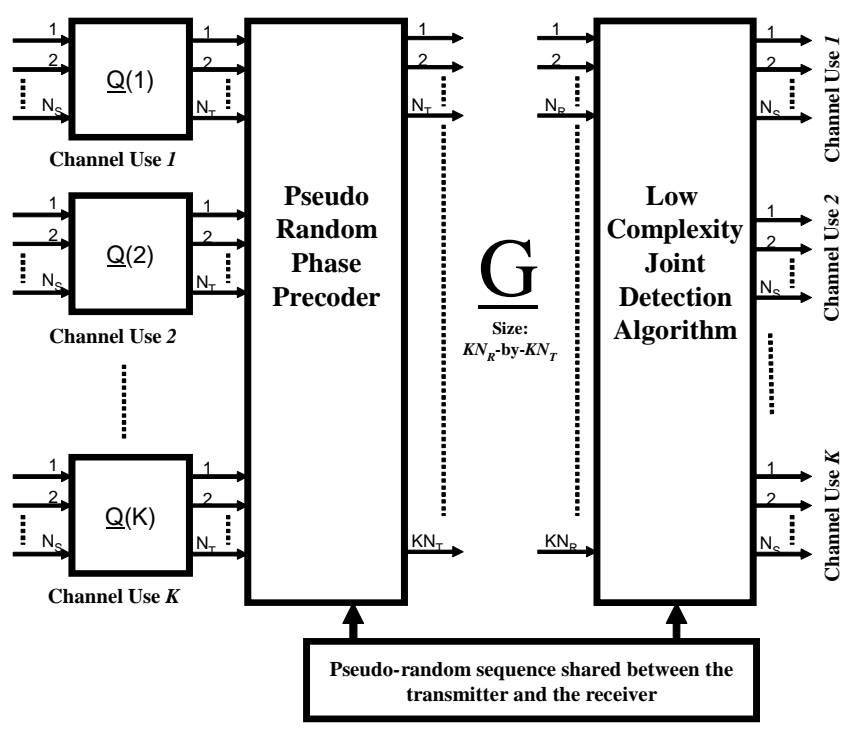

(a)

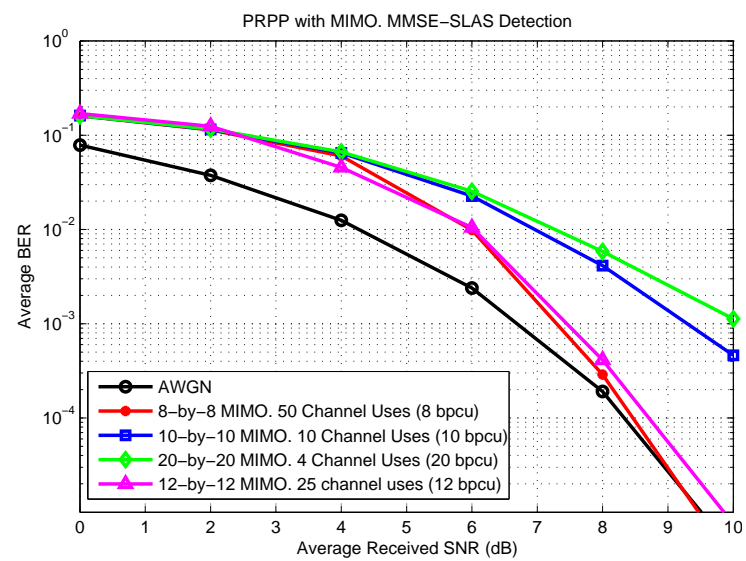

(b)

Fig. 6: MIMO transmitter with PRPP to realize multiplexing in both space and time (or frequency) is described in Fig. 6a, whereas Fig. $6 \mathrm{~b}$ presents the performance of uncoded singleuser MIMO systems with PRPP.

During the $k$ th channel use, the $N_{R}$-by-1 received signal is given by

$$
\mathbf{y}_{k}=\sqrt{\frac{P_{T}}{N_{T}}} \mathbf{H}_{k} \mathbf{x}_{k}+\mathbf{w}_{k}, \quad k=1, \ldots, K,
$$

where $P_{T}$ is the average transmitter power, $\mathbf{H}_{k}$ is the $N_{R}$-by$N_{T}$ channel matrix, $\mathbf{x}_{k}=\mathbf{Q}_{k} \mathbf{d}_{k}$ is the $N_{T}$-by-1 transmitted signal with $\mathbf{d}_{k}$ being the $N_{S}$-by-1 modulation vector, and $\mathbf{w}_{k}$ is the $N_{R}$-by-1 noise vector. 
Upon stacking $\mathbf{y}_{k}$ over $K$ channel uses, we have

$$
\begin{aligned}
\mathbf{y} & =\left[\begin{array}{c}
\mathbf{y}_{1} \\
\vdots \\
\mathbf{y}_{K}
\end{array}\right] \\
& =\sqrt{\frac{P_{T}}{N_{T}}} \operatorname{Diag}\left(\mathbf{H}_{1}, \ldots, \mathbf{H}_{K}\right)\left[\begin{array}{c}
\mathbf{x}_{1} \\
\vdots \\
\mathbf{x}_{K}
\end{array}\right]+\left[\begin{array}{c}
\mathbf{w}_{1} \\
\vdots \\
\mathbf{w}_{K}
\end{array}\right] \\
& =\sqrt{\frac{P_{T}}{N_{T}}} \underline{\mathbf{G}} \underline{\mathbf{x}}+\underline{\mathbf{w}},
\end{aligned}
$$

where $\underline{\mathbf{G}}$ is the $N_{R} K$-by- $N_{T} K$ effective channel matrix, and $\underline{\mathbf{x}}$ and $\underline{\underline{\mathbf{w}}}$ are of sizes $N_{T} K$-by- 1 and $N_{R} K$-by-1, respectively.

We propose to apply a pseudo-random phase precoder in both space (the number of transmit antennas) and time (the number of channel uses) to realize a large dimensional MIMO system. Denoting the $K N_{T}-$ by $-K N_{T}$ PRPP by $\mathbb{P}$, from (23), our PRPP-MIMO system

$$
\mathbf{y}=\sqrt{\frac{P_{T}}{N_{T}}} \underline{\mathbf{G}} \mathbb{P} \underline{\mathbf{x}}+\underline{\mathbf{w}}
$$

has $K N_{S}$ streams, $K N_{T}$ transmitter antennas and $K N_{R}$ receiver antennas.

The performance of uncoded PRPP-MIMO system with spatial multiplexing and MMSE-SLAS receiver is studied in Fig. 6b. We consider binary modulation with $N_{T}=N_{R}=N_{S}$ and $N_{S} \in\{8,10,12,12,20\}$, and with a spectral efficiency of $N_{S}$ bits per channel use (bpcu). The number of channel uses, $K$, is chosen from $\{4,10,25,50\}$. The BER performance of PRPP-MIMO, averaged over the number of streams and the number of channel uses, is plotted in Fig. $6 \mathrm{~b}$ as a function of the average received SNR $P_{T} / \sigma_{w}^{2}$. Also shown in Fig. 6b is the BER performance of the SISO AWGN channel. From Fig. 6b, we conclude that a MIMO system with a large number of transmitter and receiver antennas, in addition to being impractical, not necessarily leads to an improved performance over a MIMO system with relatively smaller number of transmitter and receiver antennas. What matters for a PRPP-MIMO system is the effective number of dimensions over which the modulation symbols are transmitted. For example, an 8-by8 MIMO system with 50 channel uses has 400 dimensions whereas a 20-by-20 MIMO system with 4 channel uses has only 80 dimensions. That is, the proposed PRPP-MIMO system can effectively offer excellent tradeoff between the number of streams and the number of channel uses needed to unleash the full potential of large-dimensional search algorithms such as LAS [9]- [15], probabilistic data association (PDA) [27][33] and Tabu search [34]- [36]. For example, instead of employing the large-dimensional search algorithms on a system with $N_{S}=N_{T}=N_{R}=600$ antennas [9], from Fig. 6b we conclude that near-exponential diversity can be achieved with a 8-by-8 MIMO system when the PRPP is applied over 50 channel uses, which we believe is within the reach of today's technologies [37].

\section{PRPP FOR MULTI-USER MIMO SYSTEMS}

In this section, we consider the application of PRPP for MU-MIMO systems. We focus on the uplink of a cellulartype setup with a single base-station and $K$ mobile users. The
BS is assumed to have $N_{B}$ receive antennas whereas each user is equipped with $N_{U}$ transmitter antennas. For simplicity, we assume that the number of streams per user is equal to $N_{U}$.

The vector-valued receiver signal at the $n$th channel use is

$$
\mathbf{y}_{n}=\sum_{k=1}^{K} \mathbf{H}_{n}(k) \mathbf{x}_{n}(k)+\mathbf{w}_{n},
$$

where $\mathbf{x}_{n}(k)$ is the $N_{U}$-by- 1 transmitted signal by the $k$ th user, $\mathbf{H}_{n}(k)$ is the $N_{B}$-by- $N_{U}$ channel between the $k$ th user and the BS, and $\mathbf{w}_{n}$ is the $N_{B}$-by-1 noise added at the BS. Upon stacking $\mathbf{y}_{n}$ over $N$ channel uses, we have

$$
\begin{aligned}
\underline{\mathbf{y}} & =\left[\begin{array}{c}
\mathbf{y}_{1} \\
\vdots \\
\mathbf{y}_{N}
\end{array}\right] \\
& =\sum_{k=1}^{K} \operatorname{Diag}\left(\mathbf{H}_{1}(k), \cdots, \mathbf{H}_{N}(k)\right)\left[\begin{array}{c}
\mathbf{x}_{1}(k) \\
\vdots \\
\mathbf{x}_{N}(k)
\end{array}\right]+\left[\begin{array}{c}
\mathbf{w}_{1} \\
\vdots \\
\mathbf{w}_{N}
\end{array}\right] \\
& =\sum_{k=1}^{K} \underline{\mathbf{H}}_{k} \underline{\mathbf{x}}(k)+\underline{\mathbf{w}},
\end{aligned}
$$

where $\underline{\mathbf{H}}_{k}$ is the $N_{B} N$-by- $N_{U} N$ block diagonal matrix, and $\underline{\mathbf{x}}(k)$ and $\underline{\mathbf{w}}(k)$ are of sizes $N_{U} N$-by- 1 and $N_{B} N$-by-1, respectively.

We propose to apply PRPP on the $k$ th user's information stream, $\underline{\mathbf{u}}(k)$, to produce $\underline{\mathbf{x}}(k)$ as

$$
\underline{\mathbf{x}}(k)=\mathbb{P}_{k} \underline{\mathbf{u}}(k),
$$

where

$$
\mathbb{P}_{k}=\frac{1}{\sqrt{N_{U} N}}\left[e^{j \theta_{l, m}^{k}}\right]_{l=1, m=1}^{N_{U} N, N_{U} N}
$$

is the $N_{U} N$-by- $N_{U} N$ PRPP matrix assigned to user $k$. Using (27) in (26), we obtain

$$
\begin{aligned}
\underline{\mathbf{y}} & =\left[\underline{\mathbf{H}}_{1}, \cdots, \underline{\mathbf{H}}_{K}\right] \operatorname{Diag}\left(\mathbb{P}_{1}, \cdots, \mathbb{P}_{K}\right)\left[\begin{array}{c}
\underline{\mathbf{u}}(1) \\
\vdots \\
\underline{\mathbf{u}}(K)
\end{array}\right]+\underline{\mathbf{w}} \\
& =\underline{\mathbf{G}} \underline{\mathbb{P}} \underline{\mathbf{u}}+\underline{\mathbf{w}},
\end{aligned}
$$

where $\underline{\mathbf{G}}$ is the $N_{B} N$-by- $N_{U} N K$ composite channel matrix, $\underline{P}$ is the $N_{U} N K$-by- $N_{U} N K$ composite block diagonal PRPP matrix, and $\underline{\mathbf{u}}$ and $\underline{\mathbf{w}}$ are of sizes $N_{U} N K$-by- 1 and $N_{B} N$-by-1, respectively.

In Fig. 7a the BER performance of uncoded MU-MIMO systems is evaluated with the proposed PRPP. For simplicity, we consider two users, each with a single stream, transmitting simultaneously to the BS with two receiver antennas on independent Rayleigh fading channels. We note that the BS assigns the PRPP matrices $\mathbb{P}_{1}$ and $\mathbb{P}_{2}$ to users 1 and 2, respectively, at the beginning of transmission, and each user independently precodes without the knowledge of the other user. Perfect CSI is assumed at the receiver. The per-user precoder size $N$ in Fig. $7 \mathrm{a}$ is varied from 40 to 200 binary symbols, and the BER of PRPP-MMSE-SLAS receiver, with 5 iterations per bit, and averaged over the users and the channel, is plotted as a function of the average received SNR per bit per user. From Fig. 7a we observe that the average BER decreases monotonically with the 


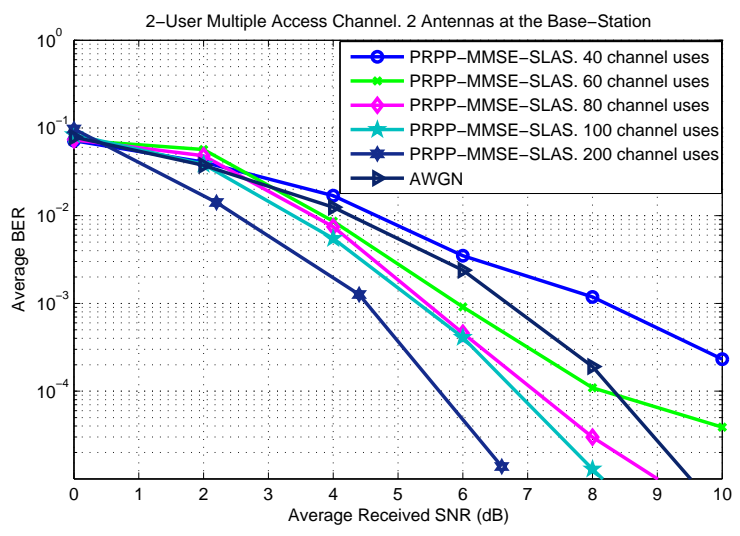

(a)

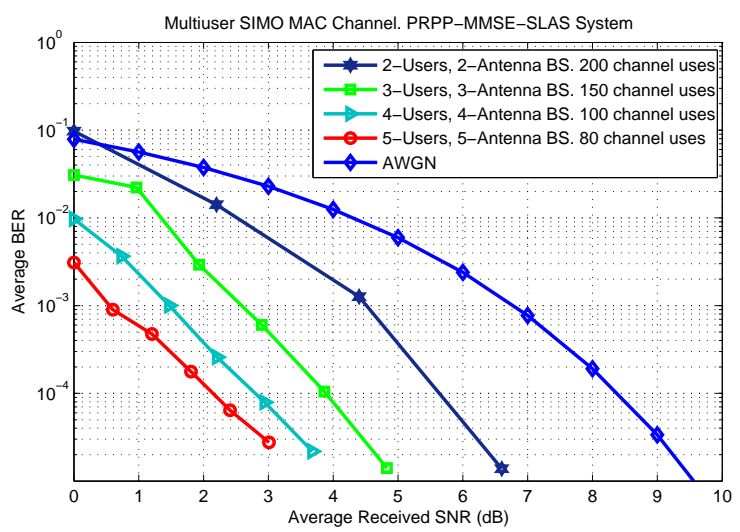

(b)

Fig. 7: Performance of PRPP applied to an uncoded $K$-user SIMO system with $N_{B}$ antennas at the base-station. Fig. 7 a shows the reliability performance as a function of the precoder size when $K=N_{B}=2$, whereas Fig. $7 \mathrm{~b}$ shows the performance for $K=N_{B} \in 2,3,4,5$ with a precoder size of 400 binary symbols.

number of channel uses over which the PRPP is applied. More importantly, with 200 channel uses, we conclude from Fig. 7 a that the performance of the proposed system at a BER of $10^{-5}$ is exactly $3.0 \mathrm{~dB}$ better than the performance of a point-to-point non-fading link with no interference. That is, in addition to completely eliminating the other-user interference, the PRPP is able to transform the fading and interference channel into a static channel, and the two receiver antennas at the BS are merely used to extract the array gain.

The impact of the number of users and the number of antennas at the BS on the BER performance of PRPP-based uncoded MU-MIMO system is investigated in Fig. 7b. Here, the number of users is increased from 2 to 5 with equal average received SNR to the BS and each user has a single stream to transmit. The number of antennas at the BS is set equal to the number of users so that the linear MMSE receiver produces an initial vector from (29). The number of channel uses $N$ for each value of $K$ is chosen in such a way that the total number of observations at the receiver, $N K$, is approximately the same for different number of users. With binary signaling, the spectral efficiency of $K$-user MU-MIMO system in Fig. $7 \mathrm{~b}$ is equal to $K$ bits per channel use. From Fig. 7b, at a BER of $10^{-5}$, we notice that the proposed PRPP-MMSE-SLAS receiver has a gain of approximately $10 \log _{10}(K) \mathrm{dB}, K=2, \ldots, 5$, over the point-to-point non-fading and inteference-free link. That is, with the PRPP applied over a modest number of channel uses, and when the number of antennas equal to the number of users, the proposed system transform the multi-user fading and interference channel into a static channel while simultaneously providing a power gain that is linear in the number of users.

\section{PRPP FOR OFDM AND SC-FDMA SYSTEMS}

We now present PRPP for uncoded OFDM and SC-FDMA waveforms. For simplicity, we consider a single user system with one transmitter antenna. Following the models in earlier sections, it is straightforward to extend this basic configuration to multiple users and multiple streams per user.

Our OFDM system description is as follows. The OFDM system bandwidth is $W \mathrm{~Hz}$, the sampling frequency is $F_{S}$ samples per second, the number of available subcarriers $N_{F F T}$, and the number of used subcarriers is $N_{S}$. The subcarrier spacing is chosen in such a way that inter-carrier interference is minimized and the cyclic prefix is chosen in such a way that inter-symbol interference is minimized.

Without loss of generality, we assume uplink transmission from an MS to a BS, and the BS is equipped with $M_{B}$ antennas for reception. Considering a tapped delay-line channel model, the frequency-domain channel between MS and BS on the $l$ th antenna, and on subcarrier $f_{k}$, is

$$
H_{l, k}=\sum_{n=1}^{L_{P}} h_{l, n} e^{-j 2 \pi \tau_{n} f_{k}}, k=1, \ldots, N_{S},
$$

where, for $l=1, \ldots, N_{B}, h_{l, n}$ is the complex-valued channel gain at a delay $\tau_{n}$ on antenna $l$ and $L_{P}$ is the number of time taps.

With $X_{k}$ denoting the symbol transmitted on subcarrier $k$, the received signal on $k$ th subcarrier on antenna $l$ is

$$
Y_{l, k}=H_{l, k} X_{k}+W_{l, k},
$$

where $W_{l, k}$ is the additive noise. Upon denoting $\mathbf{X}=\left[X_{1}, \ldots, X_{N_{S}}\right]^{T}, \quad \mathbf{Y}_{l}=\left[Y_{l, 1}, \ldots, Y_{l, N_{S}}\right]_{T}^{T}$, $\mathbf{H}_{l}=\operatorname{Diag}\left(H_{l, 1}, \ldots, H_{l, N_{S}}\right)$, and $\mathbf{W}_{l}=\left[W_{l, 1}, \ldots, W_{l, N_{S}}\right]^{T}$, the vector-valued received over $N_{B}$ antennas is

$$
\begin{aligned}
\mathbf{Y} & =\left[\begin{array}{c}
\mathbf{Y}_{1} \\
\vdots \\
\mathbf{Y}_{N_{B}}
\end{array}\right] \\
& =\left[\begin{array}{c}
\mathbf{H}_{1} \\
\vdots \\
\mathbf{H}_{N_{B}}
\end{array}\right] \mathbf{X}+\left[\begin{array}{c}
\mathbf{W}_{1} \\
\vdots \\
\mathbf{W}_{N_{B}}
\end{array}\right] \\
& =\mathbf{H X}+\mathbf{W} \\
& =\mathbf{H} \mathbb{P} \mathbf{U}+\mathbf{W},
\end{aligned}
$$

where the dimensions of $\mathbf{Y}, \mathbf{H}$ and $\mathbf{W}$ are $N_{B} N_{S}$-by-1, $N_{B} N_{S}$-by- $N_{S}$ and $N_{B} N_{S}$-by-1, respectively. $\mathbf{U}$ is the $N_{S}$-by1 subcarrier modulation vector and $\mathbb{P}$ is the $N_{S}$-by- $N_{S}$ PRPP matrix.

We can also generalize (32) to accommodate multiple PRPP blocks within a single OFDM symbol, as illustrated in Fig. 8 . 


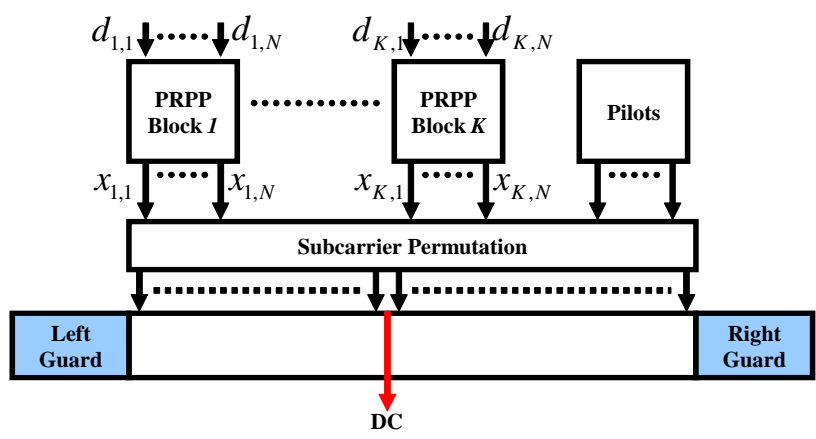

Fig. 8: OFDM transmitter with PRPP to realize multiplexing in frequency. Here, $K$ denotes the number of PRPP blocks and $\left\{d_{k, 1}, \ldots, d_{k, N}\right\}$ denotes the $N$-length modulation symbol vector that is applied to $k$ th precoder. Subcarrier permutation is applied to the precoded symbols and pilot symbols for channel estimation.

The number of used subcarriers is divided into a number of pilot subcarriers, $N_{U, P}$, for channel estimation and the remaining subcarriers, $N_{U, D}=N_{U}-N_{U, P}$, for data transmission. With a PRPP block of size $N$ subcarriers, the number of PRPP blocks within the OFDM symbol is $K=N_{U, D} / N$. The input to the $k$ th pseudo-random precoder $\mathbb{P}_{k}$ is denoted by $\mathbf{d}_{k}=\left[d_{k, 1}, \ldots, d_{k, N}\right]^{T}$ and the corresponding output is denoted by $\mathbf{x}_{k}=\left[x_{k, 1}, \ldots, x_{k, N}\right]^{T}$. The optional subcarrier permutation unit in Fig. 8 interleaves the precoder's output to extract channel frequency selectivity. Using this, (32) can be modified as

$$
\mathbf{Y}_{k}=\mathbf{G}_{k} \mathbb{P}_{k} \mathbf{d}_{k}+\mathbf{W}_{k}, \quad k=1, \ldots, K,
$$

where $\mathbf{G}_{k}$ is the effective channel of the $k$ th precoder and is of size $N_{B} N$-by- $N$.

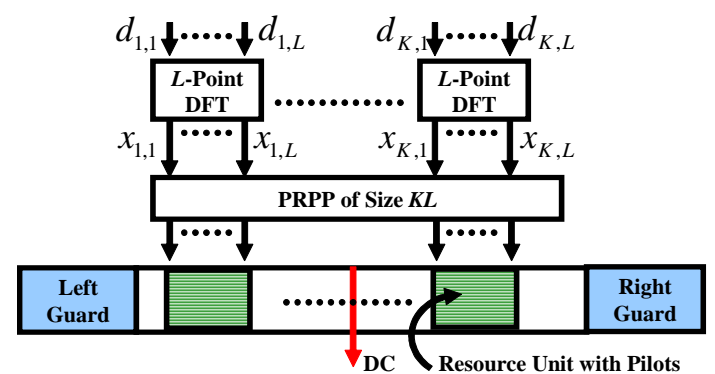

Fig. 9: SC-FDMA transmitter with PRPP to realize multiplexing in frequency while preserving the peak-to-average power ratio advantages of conventional SC-FDMA. Here, $L$ denotes the size of the DFT precoder [16] and $\left\{d_{k, 1}, \ldots, d_{k, L}\right\}$ denotes the $L$-length modulation symbol vector that is applied to the $k$ th DFT precoder. The outputs of $K$ DFT precoders are combined prior to the application of PRPP of size $K L$. By setting $\mathbb{P}=\mathbf{I}_{K L}$, we obtain the conventional SC-FDMA system.

Similar to Fig. 8, an SC-FDMA system with PRPP is shown in Fig. 9. Unlike the OFDM system in Fig. 8, in SCFDMA system DFT (Discrete Fourier Transform) precoded modulation symbols are applied as the input to the PRPP block to reduce the peak-to-average power ratio. The user's subcarrier modulation are divided into $K$ groups with $L$ modulation symbols per group. The DFT precoder is denoted by the $L$-by$L$ matrix $\mathbb{F}$. For the $k$ th group, the output of the DFT precoder is

$$
\mathbf{x}_{k}=\mathbb{F} \mathbf{d}_{k}, \quad k=1, \ldots, K,
$$

where $\mathbf{x}_{k}=\left[x_{k, 1}, \ldots, x_{k, L}\right]^{T}$ and $\mathbf{d}_{k}=\left[d_{k, 1}, \ldots, d_{k, L}\right]^{T}$. The output of the PRPP is then

$$
\mathbf{X}=\mathbb{P} \mathbf{x},
$$

where $\mathbf{x}=\left[\mathbf{x}_{1}^{T}, \ldots, \mathbf{x}_{K}^{T}\right]^{T}$, and $\mathbb{P}$ is of size $L K$-by- $L K$. Similar to (32), the received signal over $N_{B}$ antennas at the BS is

$$
\begin{aligned}
\mathbf{Y} & =\left[\begin{array}{c}
\mathbf{H}_{1} \\
\vdots \\
\mathbf{H}_{N_{B}}
\end{array}\right] \mathbb{P D i a g}(\underbrace{\mathbb{F}, \cdots, \mathbb{F}}_{K \text { times }})\left[\begin{array}{c}
\mathbf{d}_{1} \\
\vdots \\
\mathbf{d}_{K}
\end{array}\right]+\left[\begin{array}{c}
\mathbf{W}_{1} \\
\vdots \\
\mathbf{W}_{N_{B}}
\end{array}\right] \\
& =\mathbf{H} \mathbb{P} \mathbf{x}+\mathbf{W} .
\end{aligned}
$$

By setting $\mathbb{P}_{n}=\mathbf{I}_{N}$ in Fig. 8 , where $\mathbb{P}_{n}$ is the PRPP matrix for data symbols $\left\{d_{n, 1}, \ldots, d_{n, N}\right\}$, and $\mathbb{P}=\mathbf{I}_{K L}$ in Fig. 9, the proposed systems degenerate to conventional OFDM and SC-FDMA systems, respectively [16]. It is also worth mentioning that PRPP based MIMO-OFDM and MIMO-SCFDMA systems can be realized by appropriately combining the systems depicted in Figs. 6a and 8, and Figs. 6a and 9, respectively.

TABLE I: Power-delay profile. ITU Pedestrian-B channel model.

\begin{tabular}{|c|c|c|c|c|c|c|}
\hline \hline Delay (nsec) & 0 & 200 & 800 & 1200 & 2300 & 3700 \\
\hline Relative Power $(\mathrm{dB})$ & 0 & -0.9 & -4.9 & -8.0 & -7.8 & -23.9 \\
\hline \hline
\end{tabular}

The performance of PRPP-OFDM system of Fig. 8 and PRPP-SC-FDMA system of Fig. 9 is investigated in Fig. 10. Here, we consider a $10 \mathrm{MHz}$ channel bandwidth with 1024 subcarriers. The sampling frequency is $11.2 \mathrm{Msps}$, the subcarrier spacing is $10.9375 \mathrm{KHz}$, the cyclic prefix length is 128 samples, the number of left-guard subcarriers is 92 , and the number of right-guard subcarriers is 91 . Ignoring DC subcarrier, the number of useful subcarriers is 840 . The average BER performance of PRPP-OFDM system operating over the ITU Pedestrain-B (ITU-Ped-B) channel is shown in Fig. 10a. The power-delay profile of ITU-Ped-B channel is given in Table I [16]. For simplicity, in Fig. 10a we assume perfect CSI at the receiver and random permutation of modulation symbols onto the subcarriers. With one receiver antenna, as predicted by (4), the No-PRPP-MLD system has a first order diversity performance whereas the PRPP system with $N=96$ symbols has much better performance, as evidenced by the slope of its BER curve. We also study the effect of receive antenna diversity in Fig. 10a. With two receive antennas, and assuming spatially independent fading, the No-PRPP-MLD system obtains only second order diversity. On the other hand, at a BER of $10^{-4}$, the PRPP system with two receive antennas is better than the SISO AWGN performance by $0.5 \mathrm{~dB}$ (or away from the $1-$ by -2 AWGN performance by $3-0.5=2.5$ $\mathrm{dB})$. Comparing the MFB with two receiver antennas, which 


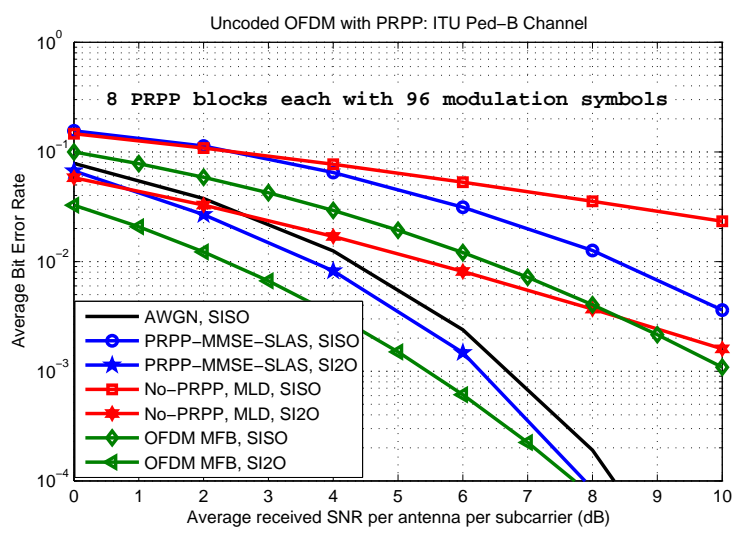

(a)

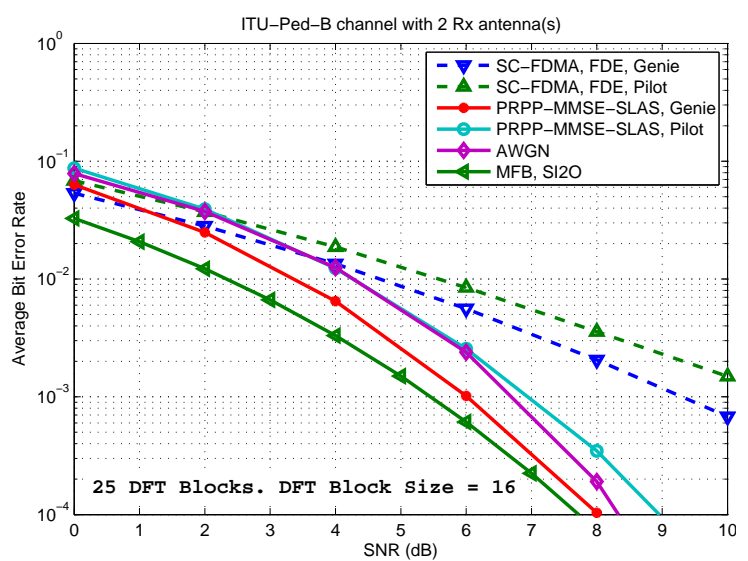

(b)

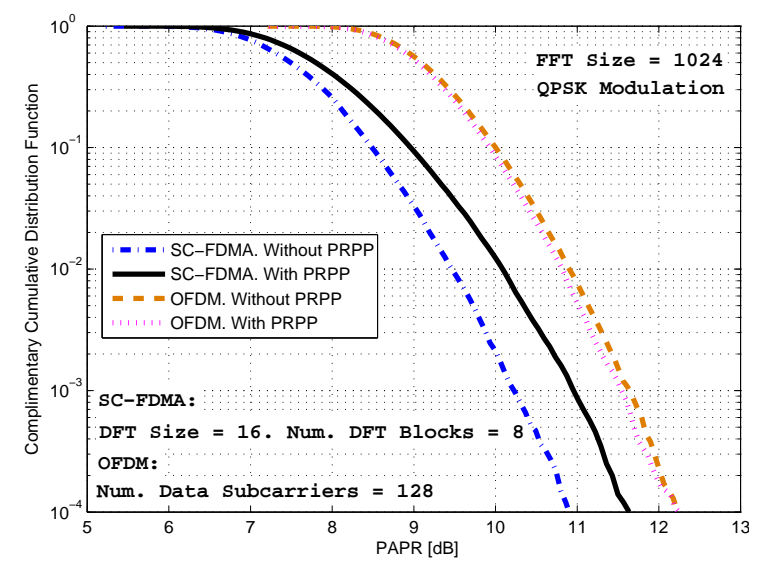

(c)

Fig. 10: Performance of PRPP for OFDM on ITU-Ped-B channel is shown in Fig. 10a. Performance of PRPP for SC-FDMA on ITU-Ped-B channel with two receiver antennas is shown in Fig. 10b. Also shown in Fig. 10b are the performances of conventional SC-FDAM with frequency-domain linear MMSE receiver. The Impact of PRPP on the peak-to-average power ratio of OFDM and SC-FDMA systems is shown in Fig. 10c.

is computed with the help of [18], we see that our system is within $0.1 \mathrm{~dB}$ of the MFB.

BER performance of SC-FDMA with PRPP is shown in Fig. 10b considering both genie-aided and pilot-aided channel estimation on ITU-Ped-B channel with two antennas at the receiver and spatially independent fading. The number of DFT blocks is set to $K=25$ and the DFT precoder size is $L=16$ modulation symbols. Also shown in Fig. 10b are the performance of SC-FDMA without PRPP when frequencydomain linear MMSE equalization (FDE) is employed (which requires inversion of a $L$-by- $L$ matrix), the AWGN reference curve, and the MFB of [18]. For channel estimation, a total of 60 pilot tones are uniformly distributed within the useful 840 subcarriers, and the $L K=16 \times 25=400$ data subcarriers are randomly loaded onto the remaining $840-60=780$ subcarriers. The pilot SNR is set to $9 \mathrm{~dB}$ above the data SNR. A simple least-squares channel estimation is considered to estimate the channel on pilot tones, and the channel on the data subcarriers is estimated by linearly interpolating the channel estimates on the pilot tones. From Fig. 10b, with perfect CSI at the receiver, we notice that with two receiver antennas SC-FDMA system without PRPP and FDE receiver yields second order diversity whereas the application of PRPP in conjunction with MMSE-SLAS receiver yields exponentially decaying error performance. For example, at a BER of $10^{-4}$, our system has $0.3 \mathrm{~dB}$ improvement over the SISO AWGN performance (or away from the 1-by-2 AWGN performance by $3-0.3=2.7 \mathrm{~dB}$ ), and is just $0.3 \mathrm{~dB}$ away from the MFB. At this BER target, channel estimation errors lead to a loss in PRPP based MMSE-SLAS receiver by approximately $1.0 \mathrm{~dB}$.

In Fig. 10c, we assess the impact of PRPP on the peakto-average power ratio (PAPR) of OFDM and SC-FDMA systems. Here, we consider QPSK subcarrier modulation. For SC-FDMA system, the DFT size is $L=16$ and the number of DFT blocks is $K=8$. For OFDM system, the payload size is $L \times K=128$ subcarriers. Whether PRPP is applied or not, the modulation symbols are randomly mapped onto the data subcarriers. A total of $10^{5}$ simulation runs are performed. From Fig. 10c, we observe that application of PRPP for SCFDMA systems leads to less than $1.0 \mathrm{~dB}$ degradation in PAPR performance (with 99 percent probability), whereas PRPP does not impact the PAPR performance of OFDM systems.

\section{CONCLUding Remarks}

We proposed a novel PRPP-based temporal multiplexing scheme for uncoded transmissions over SISO fading channels without CSI at the transmitter. With 400 binary modulation symbols and an MMSE-SLAS receiver with 5 iterations per bit, we have demonstrated that the proposed system achieves performance within $0.1 \mathrm{~dB}$ of the AWGN channel at a bit error rate of $10^{-5}$, and is robust to channel correlation and channel estimation errors. The proposed system was extended to uncoded multi-user MIMO systems where it was shown to eliminate fading and interference while simultaneously yielding maximum possible power gain. With uncoded OFDM and SC-FDMA systems, we have shown that performance within $0.3 \mathrm{~dB}$ of the MFB can be realized with as few as two receiver antennas.

Although this paper was focused on uncoded binary transmission, application of the proposed PRPP approach for multilevel modulations with channel coding and soft-decision decoding, and also with other near-ML detection schemes such as PDA [27], [28], [29], [30], [31], [32], [33] and Tabu search 
algorithms [34], [35], [36] is our on-going research work.

\section{ACKNOWLEDGMENT}

The authors thank their colleagues Drs. Simon Pun and Raymond Yim for valuable discussions, and Dr. Jinyun Zhang for encouragement and support.

\section{REFERENCES}

[1] E. Biglieri, J. G. Proakis and S. Shamai, "Fading channels: Informationtheoretic and communications aspects," IEEE Trans. Info. Theory, vol. 44. Oct. 1998, pp. 2619-2692.

[2] J. G. Proakis, Digital Communications, Fourth Edition, McGraw-Hill, 2001.

[3] M. K. Simon and M. -S. Alouini, Digital Communications over Generalized Fading Channels: A Unified Approach to Performance Analysis, Wiley \& Sons, Inc., 2000.

[4] Y. H. Lee and Y. Bar-Ness, "Power adaptation for BPSK signaling with average and peak power constraints in Rayleigh fading channels," IEEE Tran. on Comm., vol. 51, no. 11, Nov. 2003, pp. 1871-1876.

[5] V. Sharma, K. Premkumar and R. N. Swamy, "Exponential diversity achieving spatio-temporal power allocation scheme for fading channels," IEEE Trans. Info. Theory, vol. 54, no. 1, Jan. 2008.

[6] M. Schwartz, W. R. Bennett and S. Stein, Communication Systems and Techniques, New York; McGraw-Hill, 1966.

[7] R. Johannesson and K. Zigangirov, Fundamentals of Convolutional Coding, IEEE Press, 2001.

[8] J. B. Anderson and A. Svensson, Coded Modulation Systems, First Edition, Springer, 2003.

[9] K. V. Vardhan, S. K. Mohammed, A. Chockalingam, and B. S. Rajan, "A low-complexity detector for large MIMO systems and multicarrier CDMA systems," IEEE Journal on Selected Areas in Comm., vol. 26, no. 3, April 2008, pp. 473-485.

[10] S. K. Mohammed, A. Chockalingam and B. S. Rajan, "Asymptotic analysis of the performance of LAS algorithm for large MIMO detection," in Proc. IEEE PIMRC 2008.

[11] Y. T. Zhou, R. Chellappa, A. Vaid and B. K. Jenkins, "Image restoration using a neural network," IEEE Trans. Acou. Speech and Signal Processing, July 1988.

[12] Y. Sun, J.-G. Li, S.-Y. Yu, "Improvement on the performance of modified Hopfield neural network for image restoration," IEEE Trans. Image Processing, May 1995.

[13] Y. Sun, "Hopfield neural network based algorithms for image restoration and reconstruction: Part-I: Algorithms and simulations, Part-II: Performance analysis," IEEE Trans. Signal Processing, July 2000.

[14] Y. Sun, "Eliminating-highest error and fastest-metric-descent criteria and iterative algorithms for bit synchronous CDMA multiuser detection," in Proc. IEEE ICC 98.

[15] Y. Sun, "A family of linear complexity likelihood ascent search detectors for CDMA multiuser detection," in Proc. IEEE ISSSTA, Sept. 2000.

[16] F. Khan, LTE for 4G Mobile Broadband: Air Interface Technologies and Performance, Cambridge University Press, May 2009.

[17] V. Kuhn, Wireless Communications over MIMO Channels: Applications to CDMA and Multiple Antenna Systems, John Wiley \& Sons, 2006.

[18] R. Visoz and E. Bejjani, "Matched filter bound for multichannel diversity over frequency-selective Rayleigh-fading mobile channels," IEEE Trans. Veh. Technology, vol. 49, no. 5, Sept. 2000, 1832-1845.
[19] H. Hadinejad-Mahram, D. Dahlhaus, and D. Blomker, "Matched-filter bound for binary signaling over dispersive fading channels with receive diversity," IEEE Trans. Communications, vol. 52, no. 12, Dec. 2004, pp. 2082-2086.

[20] A. M. Tonello, "Performance limits for filtered multitone modulation in fading channels," IEEE Trans. Wireless Communications, vol. 4, no. 5, Sept. 2005, pp. 2121-2135.

[21] D. Rainish, "Diversity transform for fading channels", lEEE Tran. Communications, vol. 44, no. 12, Dec. 1996, pp. 1653 - 1661.

[22] J. Boutros and E. Viterbo, "Signal space diversity: a power-and bandwidth-efficient diversity technique for the Rayleigh fading channel," IEEE Tran. Information Theory, vol. 44, no. 4, July 1998, pp. 1453 1467.

[23] Y. Li, C. N. Georghiades, and G. Huang, "Transmit diversity Over quasi-static fading channels using multiple antennas and random signal mapping," IEEE Tran. Communications, vol. 51, no. 11, Nov. 2003, pp. 1918-1926.

[24] J. Jalden, B. Ottersten, "On the complexity of sphere decoding in digital communications," IEEE. Trans. Signal Processing, vol. 53, no. 4, April 2005, pp. 1474-1484.

[25] G. H. Golub and C. F. Van Loan , Matrix Computations, Third Edition, The Johns Hopkins University Press, 1996.

[26] F. Vincent and O. Besson, "Steering vector errors and diagonal loading," IEE Proc., Radar Sonar Navig., vol. 151, no. 6, Dec. 2004, pp. 337-343.

[27] J. Luo, K. Pattipati, P. Willett and F. Hasegawa, "Near-optimal multiuser detection in synchronous CDMA using probabilistic data association," IEEE Communications Letters, vol. 5, no. 9, pp. 361-363, Sept. 2001.

[28] Y. Huang and J. Zhang, "Generalized probabilistic data association multiuser detection," in Proc. IEEE ICC'2004, June-July 2004.

[29] P. H. Tan and L. K. Rasmussen, "Multiuser detection in CDMA-A comparison of relaxation, exact and heuristic search methods," IEEE Transactions on Wireless Communications, vol. 3, no. 5, pp. 1802-1809, Sept. 2004.

[30] D. Pham, K. Pattipati, P. Willett and J. Luo, "A generalized probabilistic data association detector for multi antenna systems," IEEE Communications Letters, vol. 8, no. 4, pp. 205-207, April 2004.

[31] P. H. Tan and L. K. Rasmussen, "Asymptotically optimal nonlinear MMSE multiuser detection based on multivariate Gaussian approximation," IEEE Transactions on Communications, vol. 54, no. 8, pp. 14271438, Aug. 2006.

[32] Y. Jia, C. M. Vithanage, C. Andreiu and R. J. Piechocki, "Probabilistic data association for symbol detection in MIMO systems," IEE Electronic Letters, vol. 42, no. 1, 05 Jan 2006.

[33] S. K. Mohammed, A. Chockalingam and B. S. Rajan, "Low-complexity near-ML decoding of large non-orthogonal STBCs using PDA". Availabe at http://arxiv.org/abs/0901.1869.

[34] F. Glover and M. Laguna, Tabu Search, Kluwer Academic Publishers, 1997.

[35] H. Zhao, L. Tong and W. Wang, "Tabu search detection for MIMO systems," in Proc. IEEE PIMRC'2007, Sept. 2007.

[36] N. Srinidhi, S. K. Mohammed, A. Chockalingam and B. Sunder Rajan, "Low-complexity near-ML decoding of large non-orthogonal STBCs using reactive tabu search," in Proc. IEEE ISIT'2009, June-July 2009.

[37] D. Kuhling, A. Ibing and V. Jungnickel, " $12 \times 12$ MIMO-OFDM realtime implementation for 3GPP LTE + on a cell processor," in Proc. 14th European Wireless Conference, June 2008. 